\title{
The method of fundamental solutions for inverse obstacle acoustic scattering
}

\author{
A. Karageorghis ${ }^{1} \&$ D. Lesnic ${ }^{2}$ \\ ${ }^{1}$ Department of Mathematics and Statistics, University of Nicosia, Cyprus \\ ${ }^{2}$ Department of Applied Mathematics, University of Leeds, UK
}

\begin{abstract}
In this paper we propose a simple method for detecting (shape, size and location) a scatterer embedded in a host acoustic homogeneous medium from scant measurements of the scattered acoustic pressure in the vicinity (near-or far-field) of the obstacle. We develop a nonlinear constrained minimization regularized method of fundamental solutions for obtaining the numerical solution of the inverse problem. The stability of the numerical scheme is investigated by inverting measurements contaminated with noise.
\end{abstract}

Keywords: inverse acoustic scattering, method of fundamental solutions.

\section{Introduction}

The inverse problems of time-harmonic acoustic scattering of waves from obstacles of arbitrary shape embedded in a fluid, or solid medium have been of considerable interest to researchers for many years, see e.g. [1]. In addition to being of academic interest, these problems have physical applications in the fields of radar and sonar detection; the ability to "see" in real time in complete darkness in murky water for deep sea submarines, underwater surveillance and target acquisition, detection of objects in the ocean, either fully submerged or partially buried in the seafloor, ultrasound medical imaging of soft tissues, nondestructive testing of materials, etc.

The inverse problem we consider in this paper is to determine the boundary of an insonified scatterer from scant measurements of its response when excited by impinging plane waves. The scatterer can be sound-soft, sound-hard or convectively embedded in the full-space. Although some uniqueness results are known, notably in [2], the problem is still difficult to solve since it is nonlinear, 
ill-posed (unstable), and computationally intensive in the high-frequency regime, see [3].

Integral equation methods, see e.g. [4], provide a basic tool in scattering theory mainly due to the fact that the formulation of obstacle scattering problems leads to boundary value problems defined over unbounded domains. Hence, their formulation in terms of boundary integral equations not only reduces by one the dimensionality of the problem, but also replaces a problem over an unbounded domain by one over a bounded contour, or surface. From a numerical point of view both these advantages have made integral equation methods, such as the boundary element method (BEM) one of the most powerful technique for the approximate solution of obstacle scattering in homogeneous media, see [5].

The method of fundamental solutions (MFS) may be viewed as a meshless BEM in which the governing field is approximated by a linear combination of fundamental solutions for the Helmholtz equation whose singularities are placed outside the solution domain. In contrast to the BEM, the MFS provides accurate approximations directly at points arbitrarily close to the boundary. Moreover, since no shape functions or elements are involved, it is easier to implement than the BEM. Also, the fictitious eigenfrequency difficulty with occurs with the BEM for the exterior Helmholtz problem apparently is not present in the MFS, [6]. Up to now, the MFS has never been applied to inverse obstacle scattering, although its principal idea of representing the solution as a single-layer potential has been used previously in [7].

In this paper, first, the direct, linear and well-posed problem of acoustic scattering for time-harmonic waves from impenetrable obstacles is approached using the MFS to find the scattered wave. Then, using a random noisy perturbation of the scattered wave as input data, the inverse, nonlinear and ill-posed problem of determining the boundary of the obstacle is approached based on a regularized optimization procedure which uses the MFS solver at each iteration until a prescribed stopping criterion is satisfied. A similar approach using the BEM instead of MFS has been previously investigated in [8], but only for sound-hard obstacles parameterised by a maximum of seven parameters and for exact scattered wave data only.

The method proposed in this paper can be easily extended to penetrable obstacles, as well as to electromagnetic scattering problems.

\section{The direct problem}

We are concerned with the scattering of a time harmonic acoustic wave by a bounded impenetrable obstacle $D \subset \mathbb{R}^{n}$. The wave motion can then be determined from the potential $u$ which satisfies the Helmholtz equation

$$
\Delta u+k^{2} u=0 \quad \text { in } \quad \mathbb{R}^{n} \backslash \bar{D},
$$

where $k>0$ is the wave number.

For the direct (forward) scattering problem, we are given the wave number $k>0$, a bounded region $D \subset \mathbb{R}^{n}$ with boundary $\partial D \in C^{2}$ such that $\mathbb{R}^{n} \backslash \bar{D}$ is 
connected, and an incident field given by a plane wave moving in the unit direction $\widehat{d}$, namely

$$
u^{\mathrm{inc}}(\boldsymbol{x})=\mathrm{e}^{i k \boldsymbol{x} \cdot \widehat{\boldsymbol{d}}} .
$$

Then the scattering of $u^{\text {inc }}$ by $D$ produces a scattered wave $u^{\mathrm{s}}$ with corresponding total field $u=u^{\mathrm{s}}+u^{\text {inc }}$. The direct scattering problem is to determine the scattered pressure $u^{\mathrm{s}} \in C^{2}\left(\mathbb{R}^{n} \backslash \bar{D}\right) \cap C^{1}\left(\mathbb{R}^{n} \backslash D\right)$ which satisfies the Helmholtz equation (1), i.e.

$$
\Delta u^{\mathrm{s}}(\boldsymbol{x})+k^{2} u^{\mathrm{s}}(\boldsymbol{x})=0, \quad \boldsymbol{x} \in \mathbb{R}^{n} \backslash \bar{D},
$$

the Sommerfeld infinity radiation condition

$$
\lim _{R \rightarrow \infty} R^{(n-1) / 2}\left(\frac{\partial u^{\mathrm{s}}}{\partial R}(\boldsymbol{x})-i k u^{\mathrm{s}}(\boldsymbol{x})\right)=0
$$

uniformly with respect to $\widehat{\boldsymbol{x}}=\boldsymbol{x} /|\boldsymbol{x}|$, where $R=|\boldsymbol{x}|$, and the boundary conditions corresponding to:

(a) a sound-soft obstacle (Dirichlet boundary condition)

$$
u(\boldsymbol{x})=0 \quad \Longleftrightarrow \quad u^{\mathrm{s}}(\boldsymbol{x})=-\mathrm{e}^{i k \boldsymbol{x} \cdot \widehat{\boldsymbol{d}}}, \quad \boldsymbol{x} \in \partial D
$$

(b) a sound-hard obstacle (Neumann boundary condition)

$$
\frac{\partial u}{\partial \nu}(\boldsymbol{x})=0 \Longleftrightarrow \frac{\partial u^{\mathrm{s}}}{\partial \nu}(\boldsymbol{x})=-\frac{\partial\left(\mathrm{e}^{i k \boldsymbol{x} \cdot \widehat{\boldsymbol{d}}}\right)}{\partial \nu}(\boldsymbol{x}), \quad \boldsymbol{x} \in \partial D,
$$

where $\boldsymbol{\nu}$ is the outward unit normal to $\partial D$.

(c) a convective obstacle (impedance, Robin boundary condition)

$$
\begin{array}{r}
\frac{\partial u}{\partial \nu}(\boldsymbol{x})+i \lambda u(\boldsymbol{x})=0, \quad \Longleftrightarrow \frac{\partial u^{\mathrm{s}}}{\partial \nu}(\boldsymbol{x})+i \lambda u^{\mathrm{s}}(\boldsymbol{x}) \\
=-\frac{\partial\left(\mathrm{e}^{i k \boldsymbol{x} \cdot \widehat{\boldsymbol{d}}}\right)}{\partial \nu}(\boldsymbol{x})-i \lambda \mathrm{e}^{i k \boldsymbol{x} \cdot \widehat{\boldsymbol{d}}}, \quad \boldsymbol{x} \in \partial D,
\end{array}
$$

where $\lambda \in \mathbb{C}, \Re(\lambda) \geq 0$ is the impedance parameter.

In the above model we have assumed that the obstacle $D$ is impenetrable. The case of penetrable obstacles which leads to a transmission problem will be investigated elsewhere.

The unique solvability of the direct problems (3)-(5), (3), (4) and (6), (3), (4) and (7) is well-established, see e.g. [1]. Furthermore, one has the following Green's representation formula

$$
\eta(\boldsymbol{x}) u^{\mathrm{s}}(\boldsymbol{x})=\int_{\partial D}\left[u^{\mathrm{s}}(\boldsymbol{y}) \frac{\partial G_{n}}{\partial \nu(\boldsymbol{y})}(\boldsymbol{x}, \boldsymbol{y})-G_{n}(\boldsymbol{x}, \boldsymbol{y}) \frac{\partial u^{\mathrm{s}}}{\partial \nu}(\boldsymbol{y})\right] d s(\boldsymbol{y}), \quad \boldsymbol{x} \in \mathbb{R}^{n} \backslash D,
$$


where $\eta(\boldsymbol{x})=1$ if $\boldsymbol{x} \in \mathbb{R}^{n} \backslash \bar{D}$ and $\eta(\boldsymbol{x})=\frac{1}{2}$ if $\boldsymbol{x} \in \partial D$, and $G_{n}$ is a fundamental solution of the Helmholtz equation defined by

$$
G_{n}(\boldsymbol{x}, \boldsymbol{y})=\left\{\begin{array}{cc}
\frac{i}{4} H_{0}^{(1)}(k|\boldsymbol{x}-\boldsymbol{y}|), & n=2, \\
\frac{\mathrm{e}^{i k|\boldsymbol{x}-\boldsymbol{y}|}}{4 \pi|\boldsymbol{x}-\boldsymbol{y}|}, & n=3,
\end{array}\right.
$$

where $H_{0}^{(1)}$ denotes the Hänkel function of the first kind and of order zero.

\section{The far-field pattern}

The radiation condition (4) leads to an asymptotic behaviour for the scattered wave of the form

$$
u^{\mathrm{S}}(\boldsymbol{x})=\frac{\mathrm{e}^{i k R}}{R^{(n-1) / 2}}\left\{u_{\infty}(\widehat{\boldsymbol{x}})+O\left(R^{-1}\right)\right\}, \quad R \rightarrow \infty
$$

uniformly with respect to $\widehat{\boldsymbol{x}}$, where the function $u_{\infty}$ is called far-field pattern of the scattered wave. The following representations for the far-field pattern are given in [9].

(a) In the case of the Dirichlet boundary condition (5) we have

$$
u_{\infty}(\widehat{\boldsymbol{x}})=-\gamma_{n} \int_{\partial D} \frac{\partial u}{\partial \nu}(\boldsymbol{y}) \mathrm{e}^{-i k \widehat{\boldsymbol{x}} \cdot \boldsymbol{y}} d s(\boldsymbol{y}) .
$$

(b) In the case of the Neumann boundary condition (6) we have

$$
u_{\infty}(\widehat{\boldsymbol{x}})=\gamma_{n} \int_{\partial D} u(\boldsymbol{y}) \frac{\partial}{\partial \nu}\left(\mathrm{e}^{-i k \widehat{\boldsymbol{x}} \cdot \boldsymbol{y}}\right) d s(\boldsymbol{y}) .
$$

(c) In the case of the Robin boundary condition (7) we have

$$
u_{\infty}(\widehat{\boldsymbol{x}})=\gamma_{n} \int_{\partial D} u(y)\left[\frac{\partial}{\partial \nu}\left(\mathrm{e}^{-i k \widehat{\boldsymbol{x}} \cdot \boldsymbol{y}}\right)+i \lambda \mathrm{e}^{-i k \widehat{\boldsymbol{x}} \cdot \boldsymbol{y}}\right] d s(\boldsymbol{y}),
$$

where $\gamma_{2}=(1+i) /(4 \sqrt{k \pi})$ and $\gamma_{3}=1 /(4 \pi)$.

\section{Inverse problems}

In this section we recall several uniqueness results for inverse obstacle scattering problems.

\section{Inverse Problem 1 (IP1)}

Determine $D$ from the knowledge of $u_{\infty}(\widehat{\boldsymbol{x}}, \widehat{\boldsymbol{d}})$ for all $\widehat{\boldsymbol{x}}, \widehat{\boldsymbol{d}} \in S^{n-1}$ and for one fixed wave number $k_{0}>0$.

Theorem 1. ([10]). Let $u^{i}$ be the total fields corresponding to sound-soft or -hard obstacles $D_{i}$ for $i=1,2$. If $u_{\infty}^{1}(\widehat{\boldsymbol{x}} ; \widehat{\boldsymbol{d}})=u_{\infty}^{2}(\widehat{\boldsymbol{x}} ; \widehat{\boldsymbol{d}})$ for all $\widehat{\boldsymbol{x}}, \widehat{\boldsymbol{d}} \in S^{n-1}$, then $D_{1}=D_{2}$. 


\section{Inverse Problem 2 (IP2)}

Determine $D$ from the knowledge of $u_{\infty}\left(\widehat{\boldsymbol{x}} ; \widehat{\boldsymbol{d}_{0}}\right)$ for all $\widehat{\boldsymbol{x}} \in S^{n-1}$, for one fixed wave number $k_{0}>0$ and for one fixed direction $\widehat{d_{0}} \in S^{n-1}$.

Theorem 2. ([2]). Let $u^{i}$ be the total fields corresponding to sound-soft obstacles $D_{i} \subset B\left(0, \zeta_{n}\right)$ for $i=1,2$, where $\zeta_{2}=2.40482 / k$ and $\zeta_{3}=\pi / k$. If $u_{\infty}^{1}(\widehat{\boldsymbol{x}})=u_{\infty}^{2}(\widehat{\boldsymbol{x}})$ for all $\widehat{\boldsymbol{x}} \in S^{n-1}$, then $D_{1}=D_{2}$.

\section{Inverse Problem 3 (IP3)}

Determine $D$ from the knowledge of $u^{\mathrm{s}}$ on some closed surface $\Gamma$ containing $D$ in its interior.

By the uniqueness for the exterior Dirichlet and Neumann problems, knowing $u^{\mathrm{s}}$ an a closed surface $\Gamma$ containing $D$ implies knowing the far-field pattern $u_{\infty}$ of $u^{\mathrm{s}}$. Therefore, the uniqueness results of Theorems 1 and 2 for the reconstruction from far-field data immediately carry over to the case of near-field data.

Although Theorems 1 and 2 ensure the uniqueness of the solution of the IP1, IP2 and IP3, the inverse problems are still ill-posed (unstable), as can be seen from the following theorem.

Theorem 3. ([7]). For a fixed incident field $u^{\text {inc }}$, the mapping $\partial D \mapsto u_{\infty}$ is continuous from $C^{1}$ into $L^{2}\left(S^{n-1}\right)$.

Therefore, one has to solve the nonlinear and ill-posed operator equation

$$
F(\partial D)=u_{\infty}
$$

for the unknown boundary $\partial D \in C^{1}$ by standard inversion methods, [5].

The IP1 has been treated in detail in [11] and therefore, in this study only the IP2 and IP3 will be investigated.

\section{The method of fundamental solutions (MFS)}

Based on the density results of Bogomolny [12], in the MFS we seek the solution of Helmholtz equation (3) in the form, see e.g. [13],

$$
u^{\mathrm{s}}(\boldsymbol{x})=\sum_{j=1}^{N} c_{j} G_{n}\left(\boldsymbol{x}, \boldsymbol{y}_{j}\right), \quad \boldsymbol{x} \in \mathbb{R}^{n} \backslash \bar{D},
$$

where $\boldsymbol{y}_{j} \in D$ are singularities located in $D$, which are assumed fixed and prescribed, and $c_{j}$ are unknown coefficients to be determined by imposing the boundary conditions (5), (6) or (7).

We consider the inverse problem IP3 for simplicity, and choose as measurements

$$
u^{\mathrm{s}}\left(\boldsymbol{x}_{m}\right)=\phi_{m}, \quad \frac{\partial u^{\mathrm{s}}}{\partial \nu}\left(\boldsymbol{x}_{m}\right)=\psi_{m}, \quad m=\overline{1, M},
$$

where $\left(\boldsymbol{x}_{m}\right)_{m=1, M}$ are uniformly distributed on the surface of the ball $B\left(\mathbf{0}, \zeta_{n}\right)$. Assuming that the obstacle $D$ is star-shaped, we also consider $N$ distributed points 
$\tilde{\boldsymbol{x}}_{m}$ on $\partial D$ characterized by the radii $r_{m}=r\left(\widehat{\tilde{\boldsymbol{x}}}_{m}\right)$ for $m=\overline{1, N}$ on which we apply the boundary conditions

$$
u^{\mathrm{s}}\left(\tilde{\boldsymbol{x}}_{m}\right)=-\mathrm{e}^{i k \tilde{\boldsymbol{x}}_{m} \cdot \widehat{\boldsymbol{d}}}
$$

or

or

$$
\frac{\partial u^{\mathrm{s}}}{\partial \nu}\left(\tilde{\boldsymbol{x}}_{m}\right)=-\frac{\partial}{\partial \nu} \mathrm{e}^{i k \tilde{\boldsymbol{x}}_{m} \cdot \widehat{\boldsymbol{d}}}
$$

$$
\frac{\partial u^{\mathrm{s}}}{\partial \nu}\left(\tilde{\boldsymbol{x}}_{m}\right)+i \lambda u^{\mathrm{s}}\left(\tilde{\boldsymbol{x}}_{m}\right)=-\frac{\partial}{\partial \nu}\left(\mathrm{e}^{i k \tilde{\boldsymbol{x}}_{m} \cdot \widehat{\boldsymbol{d}}}\right)-i \lambda \mathrm{e}^{i k \tilde{\boldsymbol{x}}_{m} \cdot \widehat{\boldsymbol{d}}} .
$$

We also choose the singularities located at the $N$ uniformly distributed points $\boldsymbol{y}_{j}$ in $D$ characterized by radii $r\left(\boldsymbol{y}_{j}\right)=\eta r_{j}, j=\overline{1, N}$, where $\eta \in(0,1)$ is an appropriately chosen constant. Since $D \subset B\left(\mathbf{0}, \zeta_{n}\right)$ this also ensures that $\boldsymbol{y}_{j}$ are not situated on a closed curve $\Gamma_{0}$ for which $-k^{2}$ could be an eigenvalue for the Dirichlet problem for the Laplace equation in the interior domain enclosed by $\Gamma_{0}$. Therefore, we have $2 N$ unknowns $\left(r_{m}\right)_{m=\overline{1, N}}$ and $\left(c_{j}\right)_{j=\overline{1, N}}$.

Imposing the sound-soft boundary condition (15) say, results in $N$ equations, namely,

$$
-\mathrm{e}^{i k \tilde{\boldsymbol{x}}_{m} \cdot \hat{\boldsymbol{d}}}=\sum_{j=1}^{N} c_{j} G_{n}\left(\tilde{\boldsymbol{x}}_{m}, \boldsymbol{y}_{j}\right), \quad m=\overline{1, N} .
$$

Imposing the measurement conditions (14) results in $2 M$ equations, namely,

$$
\phi_{m}=\sum_{j=1}^{N} c_{j} G_{n}\left(\boldsymbol{x}_{m}, \boldsymbol{y}_{j}\right), \quad \psi_{m}=\sum_{j=1}^{N} c_{j} \frac{\partial G_{n}}{\partial \nu}\left(\boldsymbol{x}_{m}, \boldsymbol{y}_{j}\right), \quad m=\overline{1, M}
$$

\subsection{Two-dimensional implementation}

The two-dimensional MFS implementation is similar to that developed by the authors in [14] for the electrical impedance tomography in electrostatics. Let $\tilde{\theta}_{m}=2 \pi m / N$ for $m=\overline{0, N}$, be a uniform discretisation of the interval $[0,2 \pi]$. Assuming that the obstacle $D$ is star-shaped with respect to the origin, we consider the unknown boundary $\partial D$ given in parametric form by the points

$$
\tilde{\boldsymbol{x}}_{m}=\left(r_{m} \cos \left(\tilde{\theta}_{m}\right), r_{m} \sin \left(\tilde{\theta}_{m}\right)\right), \quad m=\overline{1, N},
$$

where $r_{m}>0$ for $m=\overline{1, N}$ are unknown. The measurements (14) are given on the circle $B\left(\mathbf{0}, \zeta_{2}\right)$ which has the parametric form

$$
\boldsymbol{x}_{m}=\left(\zeta_{2} \cos \left(\theta_{m}\right), \zeta_{2} \sin \left(\theta_{m}\right)\right), \quad m=\overline{1, M},
$$

where $\theta_{m}=2 \pi m / M$ for $m=\overline{0, M}$. The singularities in $D$ are given by

$$
\boldsymbol{y}_{j}=\left(\eta r_{j} \cos \left(\theta_{j}\right), \eta r_{j} \sin \left(\theta_{j}\right)\right), \quad j=\overline{1, N} \text {. }
$$


Then we minimize the penalized least-squares functional

$$
\begin{gathered}
T_{\mu}(\boldsymbol{c}, \boldsymbol{r}):=\sum_{m=1}^{N}\left|\sum_{j=1}^{N} c_{j} G_{2}\left(\tilde{\boldsymbol{x}}_{m}, \boldsymbol{y}_{j}\right)+\mathrm{e}^{i k \tilde{\boldsymbol{x}}_{m} \cdot \hat{\boldsymbol{d}}}\right|^{2} \\
+\sum_{m=1}^{M}\left|\sum_{j=1}^{N} c_{j} G_{2}\left(\boldsymbol{x}_{m}, \boldsymbol{y}_{j}\right)-\phi_{m}\right|^{2}+\sum_{m=1}^{M}\left|\sum_{j=1}^{N} c_{j} \frac{\partial G_{2}}{\partial \nu}\left(\boldsymbol{x}_{m}, \boldsymbol{y}_{j}\right)-\psi_{m}\right|^{2} \\
+\mu_{1} \sum_{j=1}^{N}\left|c_{j}\right|^{2}+\quad \mu_{2} \sum_{\ell=2}^{M}\left(r_{\ell}-r_{\ell-1}\right)^{2},
\end{gathered}
$$

where $\mu_{1}, \mu_{2}>0$ are regularization parameters, subject to the simple bounds on the variables

$$
0<r_{m}<\zeta_{2}, \quad m=\overline{1, N}
$$

This optimization problem is accomplished using the MINPACK [15] routine lmdif which minimizes the unconstrained sum of squares of nonlinear functions and which does not require supplying the gradient of the functional (23). The constraints (24) are imposed during the iterative procedure by adjustment at each iteration. The minimization process terminates when either a user-specified tolerance is achieved or when a user-specified maximum number of function evaluations, maxfev, is reached. Thus in some cases, it is possible that the actual number of function evaluations preformed, $n f e v$, is less than maxfev. In all numerical experiments carried out we set the tolerance to be equal to $10^{-10}$. The initial guess is only constrained to (24) and we have taken it to be a circle of radius $r_{0}$ centered at the origin.

The calculation of the fundamental solution $G_{2}$ in (8) and its derivatives is carried out using the code MJY01A from [16].

In three-dimensions one can use spherical coordinates.

\section{Numerical results and discussion}

We consider the simple case of a plane wave of wave number $k=1$ forming an angle of $\alpha=0$ with the $x$-axis impinging on a circular sound-soft scatterer of unit radius $B(0 ; 1)$. The measurements (14) are taken on the circle of radius $\zeta_{2}=2.40428$. The exact solution for the direct scattering from a sound-soft circular obstacle $B(0 ; a)$ satisfying equations (1), (2), (4) and (5) is given by, see 
e.g., [1],

$$
\begin{array}{r}
u(r, \theta)=J_{0}(k r)-\frac{J_{0}(k a) H_{0}^{(1)}(k r)}{H_{0}^{(1)}(k a)}+ \\
2 \sum_{\ell=1}^{\infty} i^{\ell} \cos (\ell \theta)\left[J_{\ell}(k r)-\frac{J_{\ell}(k a) H_{\ell}^{(1)}(k r)}{H_{\ell}^{(1)}(k a)}\right], \\
(r, \theta) \in \mathbb{R}^{2} \backslash B(\mathbf{0} ; a), r \geq 1, \theta \in[0,2 \pi),
\end{array}
$$

where $H_{\ell}^{(1)}$ and $J_{\ell}$ are the Hänkel and Bessel functions of the first kind of order $\ell$, respectively. Note that the expression (25) is calculated by means of the routine MJYNA from [16]. We take $r_{0}=0.6$ and $\eta=0.3$. In order to investigate the stability of the numerical solution, we fix $M=N=64$ and vary the amount of noise $p$ included in the input data (14).

In Figure 1 we present the plots of the reconstructed boundary for noise $p=5 \%$, no regularization and with various numbers of $n f e v$. It can be observed that as $n f e v$ increases so does the instability. In Figure 2 we present the plots of the reconstructed boundary for various values of the regularization parameter $\mu_{1}$ in (23) for $\mu_{2}=0$ and $p=5 \%$. In all cases maxfev was set to $5 \times 10^{5}$. Interestingly, the effect of increasing the value of $\mu_{1}$ was to reduce the number of $\mathrm{nfev}$ where convergence was reached. Improved stable results were obtained for $\mu_{1}=O\left(10^{-4}\right) \div O\left(10^{-2}\right)$. In Figure 3 we present the plots of the reconstructed boundary for various values of the regularization parameter $\mu_{2}$ in (23) for $\mu_{1}=0$, $p=5 \%$ and maxfev $=5 \times 10^{5}$. Improved stable results were obtained for $\mu_{2}=O\left(10^{-2}\right) \div O(1)$.

\section{Conclusions}

In this paper, it has been shown that the MFS is well-suited for the solution of inverse obstacle problems arising in acoustic scattering. The numerical experiments yield accurate results for exact data, but instabilities appear when
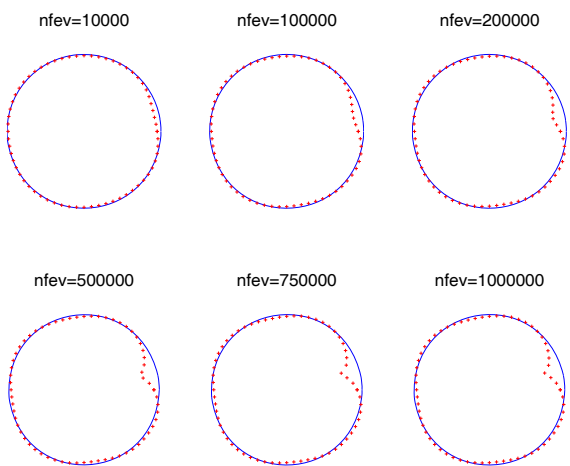

Figure 1: Results for various values of $\mathrm{nfev}$ for $p=5 \%$ and no regularization. 


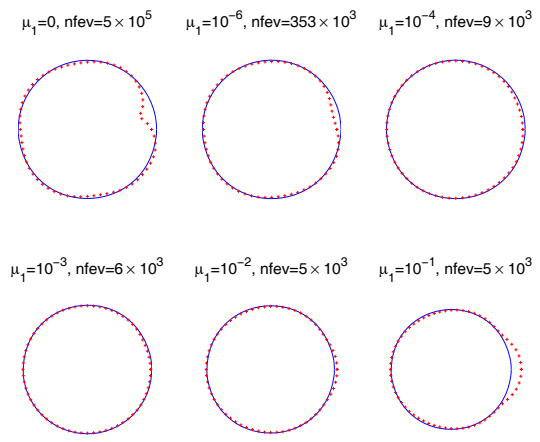

Figure 2: Results for various values of $\mu_{1}$ for $p=5 \%$, when $\mu_{2}=0$.
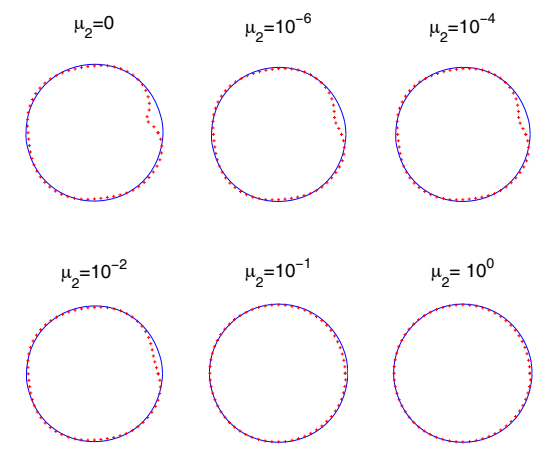

Figure 3: Results for various values of $\mu_{2}$ for $p=5 \%$, when $\mu_{1}=0$ and $\mathrm{nfev}=5 \times 10^{5}$.

noise is introduced into the input data. Regularization can be achieved either by appropriately limiting the number of function evaluations or by introducing penalty terms in the objective cost functional which is minimized. The retrieval of more complicated shapes, multiple scatterers and the implementation of a corresponding three-dimensional MFS algorithm will be the subject of future work.

\section{Acknowledgement}

The authors would like to thank the University of Cyprus for supporting this research. 


\section{References}

[1] Colton, D. \& Kress, R., Inverse acoustic and electromagnetic scattering theory, volume 93 of Applied Mathematical Sciences. Springer-Verlag: Berlin, 2nd edition, 1998.

[2] Colton, D. \& Sleeman, B.D., Uniqueness theorems for the inverse problem of acoustic scattering. IMA J Appl Math, 31(3), pp. 253-259, 1983.

[3] Kress, R., Integral equation methods in inverse acoustic and electromagnetic scattering. Boundary integral formulations for inverse analysis, Comput. Mech.: Southampton, pp. 67-92, 1997.

[4] Johansson, T. \& Sleeman, B.D., Reconstruction of an acoustically sound-soft obstacle from one incident field and the far-field pattern. IMA J Appl Math, 72(1), pp. 96-112, 2007.

[5] Kress, R., Integral equation method in inverse obstacle scattering. Eng Anal Boundary Elem, 15, pp. 171-179, 1995.

[6] Kondapalli, P., Shippy, D.J. \& G. Fairweather, G., Analysis of acoustic scattering in fluids solids by the method of fundamental solutions. $J$ Acoust Soc Am, 91, pp. 1844-1854, 1992.

[7] Kress, R. \& Zinn, A., On the numerical solution of the three-dimensional inverse obstacle scattering problem. J Comput Appl Math, 42(1), pp. 49-61, 1992.

[8] Na, S.W. \& Kallivokas, L.F., Shape detection in inverse acoustic scattering problems. 16th ASCE Engineering Mechanics Conference, University of Washington: Seattle, July 16-18, 2003.

[9] Kirsch, A., An introduction to the mathematical theory of inverse problems, volume 120 of Applied Mathematical Sciences. Springer-Verlag: New York, 1996.

[10] Kirsch, A. \& Kress, R., Uniqueness in inverse obstacle scattering. Inverse Problems, 9(2), pp. 285-299, 1993.

[11] Colton, D., Coyle, J. \& Monk, P., Recent developments in inverse acoustic scattering theory. SIAM Rev, 42(3), pp. 369-414, 2000.

[12] Bogomolny, A., Fundamental solutions method for elliptic boundary value problems. SIAM J Numer Anal, 22(4), pp. 644-669, 1985.

[13] Fairweather, G., Karageorghis, A. \& Martin, P.A., The method of fundamental solutions for scattering and radiation problems. Eng Anal Boundary Elem, 27, pp. 759-769, 2003.

[14] Karageorghis, A. \& Lesnic, D., Detection of cavities using the method of fundamental solutions. Inverse Problems Sci Eng, 17(6), pp. 803-820, 2009.

[15] Garbow, B.S., E., H.K. \& Moré, J.J., MINPACK Project. Argonne National Laboratory, 1980.

[16] Zhang, S. \& Jin, J., Computation of special functions. A Wiley-Interscience Publication, John Wiley \& Sons Inc.: New York, 1996. 